\title{
Reduced Complexity QRM-MLD for MIMO System
}

\author{
Jinyong Lee, Youngseh Kim, Kanghoon Kim, and Younglok Kim
}

\begin{abstract}
This paper proposes reduced complexity $Q R$ decomposition with M-algorithm (QRM) maximum likelihood detection (MLD) by removing superfluous paths according to the maximum squared Euclidean distance. As a result of computer simulation, the proposed method can have $56 \%$ reduction of computational complexity compare with the conventional QRM-MLD without any performance degradation at 20dB SNR. Compare with MMSE method, the $3.0 \%$ BER of conventional method is reduced to $0.04 \%$ by proposed method.
\end{abstract}

Index Terms-Channel equalization, MIMO OFDM, MMSE, QRM-MLD.

\section{INTRODUCTION}

In the next generation wireless communication system, especially 3rd generation partnership project (3GPP) long term evolution (LTE), multiple-input multiple-output (MIMO) technique is adopted in order to achieve high data throughput. Spatial multiplexing, which is one of MIMO techniques, required high performance data detection algorithm with low computational complexity. In general, maximum likelihood detection (MLD) approach has the highest performance but its computational complexity is exponentially increased by the number of transmitter antennas and the modulation level in data modem scheme [1]. Therefore QRM-MLD is used for data detection for low computational complexity [2]. However, computational complexity is not low enough in case of using QRM-MLD.

In this paper, we propose reduced complexity of the QRM-MLD which can decrease computational complexity by removing superfluous paths according to the maximum squared Euclidean distance. And we performed its bit error ratio (BER) performance by computer simulations, and compared computational complexity with minimum mean square error (MMSE) and conventional QRM-MLD methods by using real operations (ROPs).

This paper is organized as follows: The system modeling is introduced and previous detection methods are reviewed in section II. In section III, the reduced complexity QRM-MLD method is proposed. The BER performance and comparison complexity are evaluated by simulations in section IV, and the conclusion is remarked in Section $\mathrm{V}$.

\section{Signal Detection Methods}

\section{A. System Modeling}

Let $n_{t}$ is transmit antenna and $n_{r}$ is receive antenna in the

Manuscript received April 10, 2012; revised May 22, 2012.This work is supported by 'The second stage of BK21' and the CAD tool is supported by 'IDEC' in 2012.

The authors are with the Department of Electronic Engineering, Sogang University, C.P.P Box 1142, Seoul, Korea (e-mail : jiny4509@sogang.ac.kr, kyskim@sogang. ac.kr,wsockkh@sogang.ac.kr and ylkim@sogang.ac.kr) spatially multiplexed MIMO system. Then the received signal vector can be denoted by

$$
\mathbf{y}=\mathbf{H} \cdot \mathbf{x}+\mathbf{n}
$$

where $\mathbf{x}=\left[x_{1}, x_{2}, \cdots, x_{n_{t}}\right]^{\mathrm{T}}$ is the $n_{t}$ dimensional transmit vector, and $\mathbf{y}$ is the $n_{r}$ dimensional received vector, $\mathbf{n}$ is the additive white Gaussian noise (AWGN) vector. Channel matrix $\mathbf{H}$ is an $n_{t} \times n_{r}$ dimension with the element $h_{i, j}$ being the channel gain from $i$-th transmit antenna to the $j$-th receive antenna.

$$
\mathbf{H}=\left[\begin{array}{ccc}
h_{1,1} & \cdots & h_{1, n_{T}} \\
\vdots & \ddots & \vdots \\
h_{n_{R}, 1} & \cdots & h_{n_{R}, n_{T}}
\end{array}\right]=\left[\begin{array}{llll}
\mathbf{h}_{1} & \mathbf{h}_{2} & \cdots & \mathbf{h}_{n_{T}}
\end{array}\right]
$$

We assume that $\mathbf{H}$ has independently and identically distributed.

\section{B. Minimum Mean Square Error (MMSE)}

MMSE is a signal detection method considering a variance of noise which is added in receiver. It uses the weight vector $\mathbf{W}$ that minimizes the MSE value between transmitted signal $\mathbf{x}$ and estimated signal $\hat{\mathbf{x}}$. MSE value can be calculated as followings.

$$
\begin{aligned}
\varepsilon^{2} & =E\left[(\mathbf{x}-\hat{\mathbf{x}})^{H}(\mathbf{x}-\hat{\mathbf{x}})\right] \\
& =E\left[(\mathbf{x}-\mathbf{W y})^{H}(\mathbf{x}-\mathbf{W y})\right]
\end{aligned}
$$

We can obtain the optimized solution of equation (3), by this equation

$$
\mathbf{W}_{M M S E}=\left(\mathbf{H}^{H} \mathbf{H}+\sigma_{n}^{2} \mathbf{I}_{N_{T}}\right)^{-1} \mathbf{H}^{H}
$$

MMSE provides the performance degradation compared with MLD, because the weight vector $\mathbf{W}$ cannot eliminate interference signal perfectly. However it has much less computational complexity.

\section{Maximum Likelihood Detection (MLD)}

MLD is known as the optimal signal detection method for spatially multiplexed MIMO systems. MLD selects the transmitted symbol which has the smallest Euclidean distance with constellation points that transmitted symbol can be located. The decision value for estimated signal $\hat{\mathbf{x}}$ is represented as followings.

$$
\hat{\mathbf{x}}=\arg \min _{\mathbf{x}_{i} \in\left\{x_{1}, \ldots, x_{l}\right\}}\left\|\mathbf{y}-\mathbf{H} \mathbf{x}_{i}\right\|^{2}
$$


The number of $\mathbf{C}^{n_{t}}$ computational complexity is required for MLD, where $\mathbf{C}$ is the number of constellation points.

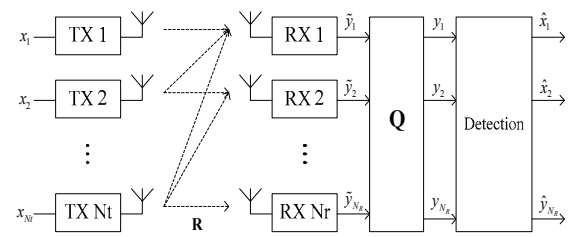

Fig. 1. Equivalent signal model using $\mathrm{QR}$ decomposition

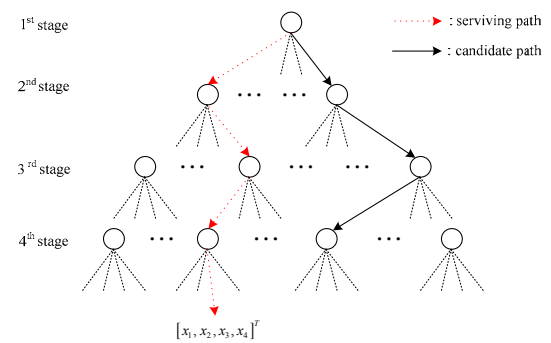

Fig. 2. Tree search based structure for QRM-MLD

\section{QR Decomposition M-Algorithm (QRM-MLD)}

QRM-MLD is the signal detection method that uses QR decomposed channel and $\mathrm{M}$ algorithm. It has less computational complexity because only $\mathrm{M}$ points that have small Euclidean distance are calculated.

Channel matrix $\mathbf{H}$ can be decomposed into unitary matrix $\mathbf{Q}$ and upper triangular matrix $\mathbf{R}$. Then received signal vector is transformed as following.

$$
\begin{array}{cr}
\tilde{\mathbf{y}}=\mathbf{Q}^{H} \mathbf{y}=\mathbf{Q}^{H} \mathbf{Q R \mathbf { R }}+\mathbf{Q}^{H} \mathbf{n}=\mathbf{R x}+\tilde{\mathbf{n}} \\
\tilde{y}_{1}=\underline{r}_{1,1} \cdot x_{1}+\underline{r}_{1,2} \cdot x_{2}+\underline{r}_{1,3} \cdot x_{3}+\underline{r}_{1,4} \cdot x_{4}+\eta_{1} \\
\tilde{y}_{2}= & \underline{r}_{2,2} \cdot x_{2}+\underline{r}_{2,3} \cdot x_{3}+\underline{r}_{2,4} \cdot x_{4}+\eta_{2} \\
\tilde{y}_{3}= & \underline{r}_{3,3} \cdot x_{3}+\underline{r}_{3,4} \cdot x_{4}+\eta_{3} \\
\tilde{y}_{4}= & \underline{r}_{4,4} \cdot x_{4}+\eta_{4}
\end{array}
$$

The equation for MLD decision value is the same as follows,

$$
\hat{\mathbf{x}}=\arg \min _{\tilde{\mathbf{x}}}\left\{\sum_{i=1}^{n_{T}}\left|\tilde{y}_{i}-\sum_{j=i}^{n_{R}} R_{i, j} \tilde{x}_{j}\right|^{2}\right\}
$$

where $\tilde{x}_{j}$ is one of $\mathrm{M}$ constellation points. Using QRM-MLD, computational complexity is reduced to $(C+M \cdot C+M \cdot$ $\mathrm{C}+\mathrm{M}$ ), when both $n_{t}$ and $n_{r}$ are four.

\section{REDUCED COMPLEXITY QRM-MLD}

We propose reduced complexity QRM-MLD which uses selected $\mathrm{M}$ paths by comparing with determined threshold value. Relation of two Euclidean distance of estimated signal using MMSE and QRM-MLD is represented by

$$
\left\|\mathbf{y}-\mathbf{H} \hat{\mathbf{x}}_{M M S E}\right\|^{2} \geq\left\|\mathbf{y}-\mathbf{H} \mathbf{x}_{Q R M-M L D}\right\|^{2}
$$

where $\tilde{\mathbf{x}}$ is selected from the constellation of each symbol. Euclidean distance of MMSE is always bigger than that of QRM-MLD, so it is set up for the maximum limit. The paths which have more accumulated matric value than the maximum limit are eliminated. Using the proposed method, we can reduce computational complexity.

The proposed algorithm is summarized as following steps : Step1: Estimate the transmit vector by multiplying $\mathbf{W}_{\text {MMSE }}$ to the received vector.

$$
\hat{\mathbf{x}}_{M M S E}=Q\left(\mathbf{W}_{M M S E} \cdot \mathbf{y}\right)
$$

Step2: Calculate Euclidean distance of estimated vector using MMSE in order to decide the maximum distance.

$$
\text { MaxDist }=\left\|\mathbf{y}-\mathbf{R} \hat{\mathbf{x}}_{M M S E}\right\|^{2}
$$

Step3: In every stage, we select $M$ paths that make the smallest accumulated squared Euclidean distances in order to avoid redundant computation. Fig. 3 explains proposed scheme.

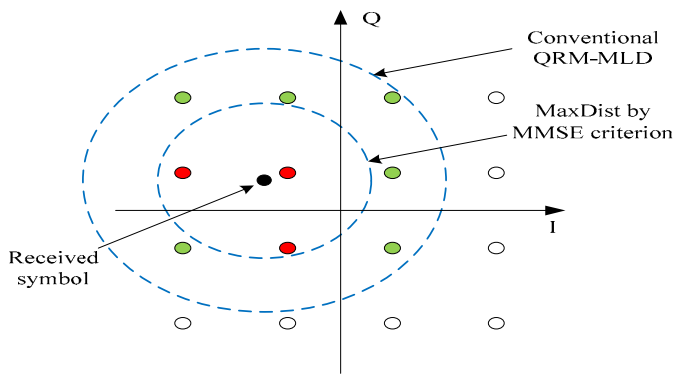

Fig. 3. Example of reduced computational QRM-MLD

\section{Simulation Results}

\section{A. Simulation Environment}

In simulation, we apply for downlink transmission in 3GPP LTE system. 16 QAM is used as the subcarrier modulation. We adopt the typical Urban 6 channel delay profile. Each channel tap is generated by an independent complex Gaussian random variable with time correlation based on Jakes' model. We assume that channel estimation is perfect. The values of some parameters are presented in Table I

TABLE I: SIMULATION PARAMETERS

\begin{tabular}{|c|c|}
\hline Parameters & value \\
\hline Transmission BW & $10 \mathrm{MHz}$ \\
\hline Sampling frequency & $15.36 \mathrm{MHz}$ \\
\hline FFT size & 1,024 \\
\hline subcarrier spacing & $15 \mathrm{kHz}$ \\
\hline Modulation & $16 \mathrm{QAM}$ \\
\hline Number of Tx antennas & 4 \\
\hline Number of Rx antennas & 4 \\
\hline Channel environment & Typical Urban6 \\
\hline Channel estimation & Perfect \\
\hline
\end{tabular}




\section{B. Performance and Computational Complexity Analysis}

In this section, we have compared the performance and computational complexity of the proposed scheme and conventional QRM-MLD. Fig. 4 shows the BER in dB scale. In simulation results, we can see that there is no performance degradation at all SNR compared with the conventional QRM-MLD.

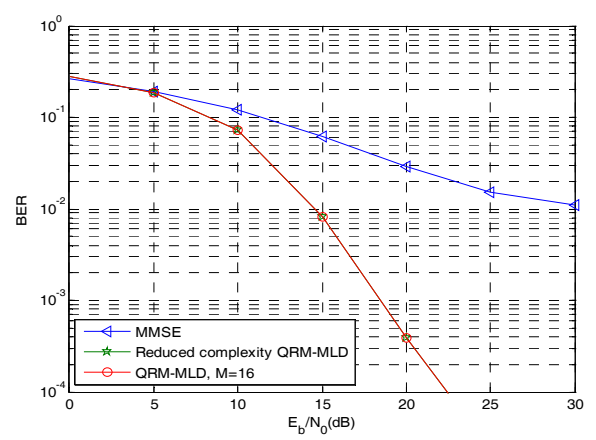

Fig. 4. BER performance of reduced complexity QRM-MLD

In order to calculate computational complexity, we define computational complexity of complex operation as shown in Table II.

In Table III, we compare the computational complexity of the signal detection scheme from the view point of the Table II. It is shown that reduced complexity QRM-MLD which reduces $59 \%$ computational complexity at $20 \mathrm{~dB}$ SNR compared with the conventional QRM-MLD.

TABLE II: COMPUTATIONAL COMPLEXITY OF COMPLEX OPERATION
\begin{tabular}{|c|c|}
\hline Complex operation & Number of real operation \\
\hline Multiplication & 4 ROP \\
\hline Division & 10 ROP \\
\hline
\end{tabular}

\section{CONCLUSION}

In this paper, we propose reduced complexity QRM-MLD which can eliminate unnecessary paths using maximum squared Euclidean distance. Simulation results have shown that the proposed scheme can achieve the same performance as conventional QRM-MLD but with smaller computational complexity.

TABLE III: RELATIVE COMPUTATIONAL COMPLEXITY OF PROPOSED SCHEME

\begin{tabular}{|c|c|c|c|}
\hline $\begin{array}{c}\text { SNR( Eb/No ) } \\
\text { Type }\end{array}$ & $15 \mathrm{~dB}$ & $20 \mathrm{~dB}$ & $\begin{array}{c}\text { Performance } \\
\text { degradation at } \\
0.1 \% \text { BER }\end{array}$ \\
\hline $\begin{array}{c}\text { QRM-MLD } \\
(\mathrm{M}=16)\end{array}$ & $100 \%$ & $100 \%$ & - \\
\hline
\end{tabular}

\begin{tabular}{|c|c|c|c|}
\hline $\begin{array}{c}\text { Reduced } \\
\text { complexity } \\
\text { QRM-MLD }\end{array}$ & $61 \%$ & $44 \%$ & - \\
\hline MMSE & $16 \%$ & $16 \%$ & $3.2 \mathrm{~dB}$ \\
\hline
\end{tabular}

\section{REFERENCES}

[1] H. Kawai, K. Higuchi, N, Maeda, and M. Sawahashi, "Adaptive Control of Surviving Symbol Replica Candidates in QRM-MLD for OFDM MIMO Multiplexing," Selected Areas in Communications, IEEE Journal on, vol. 24, no. 6, pp. 1130 - 1140, 2006.

[2] S. Nagayama and T. Hattori, "A proposal of QRM-MLD for Reduced Complexity of MLD to detect MIMO signals in Fading Environment," Vehicular Technology Conference, VTC-2006 Fall, June 2006

[3] W. Peng, S. Ma, and T. Ng, J. Wang, "Adaptive QRD-M Detection with Variable Number of Surviving Paths for MIMO Systems," Communications and Information Technologies, 2007. ISCIT '07. International Symposium on, 17-19 Oct. pp.403 - 408, 2007.

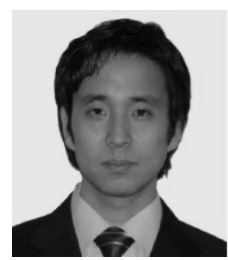

Jinyong Lee received B. S. degree in Physics and M. S. degree in Electronic Engineering from Sogang University, Seoul, Korea, in 1999 and 2007, respectively. $\mathrm{He}$ is currently working at towards the $\mathrm{Ph}$. D. degree in Electronic Engineering in Sogang University. His research interests include DSP algorithms, signal processing for communication and radar.

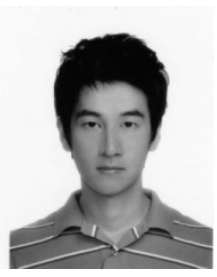

Youngseh Kim received B. S. degree in Electronic Engineering from Dankook University, Gyeonggi, Korea, in 2002 and 2009, respectively. He is currently working at towards the M. S. degree in Electronic Engineering in Sogang University. His research interests include DSP algorithms, signal processing for communication and radar.

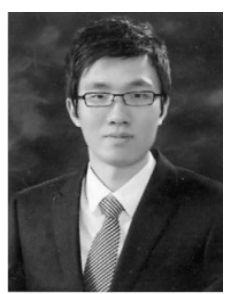

Kanghoon Kim received B. S. degree in Electronic Engineering from Hong-ik University, Seoul, Korea, in 2005 and 2011, respectively. He is currently working at towards the M. S. degree in Electronic Engineering in Sogang University. His research interests include radar signal processing and low power hardware implementation.

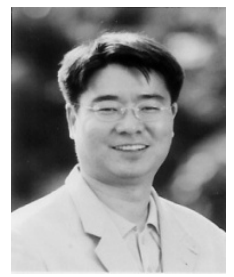

Younglok Kim received B. S. degree in Electronic Engineering from Sogang University, Seoul, Korea in 1991 and the M. S. and Ph. D. degrees in Electrical Engineering from Polytechnic Institute of NYU, Brooklyn, NY in 1993 and 1998 respectively. From 1999 to 2003, he was a senior system engineer at InterDigtal communication Corp. in Melville, NY working on $3 \mathrm{GPP}$ wireless systems. Since 2003, he joined the department of Electronic Engineering in Sogang University, where he is now an associate professor. His research interests include DSP algorithms, signal processing for communication and radar. 